\title{
Psychiatric symptoms correlate with metabolic indices in the hippocampus and cingulate in patients with mitochondrial disorders
}

\author{
RE Anglin ${ }^{1,2}$, PI Rosebush ${ }^{1}$, MD Noseworthy ${ }^{3}$, M Tarnopolsky ${ }^{4}$ and MF Mazurek ${ }^{2}$
}

There is increasing recognition that mitochondrial dysfunction may have a critical role in the pathophysiology of major psychiatric illnesses. Patients with mitochondrial disorders offer a unique window through which we can begin to understand the association between psychiatric symptoms and mitochondrial dysfunction in vivo. Using proton magnetic resonance spectroscopy ( $\left.{ }^{1} \mathrm{H}-\mathrm{MRS}\right)$, we investigated metabolic indices in mitochondrial patients in regions of the brain that have been implicated in psychiatric illness: the caudate, cingulate cortex and hippocampus. In all, 15 patients with mitochondrial disorders and 15 age- and sex-matched controls underwent a comprehensive psychiatric assessment, including the administration of standardized psychiatric rating scales, followed by single voxel ${ }^{1} \mathrm{H}$-MRS of the caudate, cingulate cortex and hippocampus to measure $\mathrm{N}$-acetyl aspartate (NAA), creatine (Cr), glycerophosphocholine (GPC), myoinositol and glutamate + glutamine (Glx). Pearson's correlation coefficients were used to determine correlations between metabolites and the psychiatric rating scales. Anxiety symptoms in these patients correlated with higher GPC, Glx, myoinositol and $\mathrm{Cr}$ in the hippocampus. Impaired level of function as a result of psychiatric symptoms correlated with higher GIx and GPC in the cingulate cortex. In summary, we found remarkably consistent, and statistically significant, correlations between anxiety and metabolic indices in the hippocampus in patients with mitochondrial disorders, while overall impairment of functioning due to psychiatric symptoms correlated with metabolic markers in the cingulate cortex. These findings lend support to the notion that mitochondrial dysfunction in specific brain regions can give rise to psychiatric symptoms. In particular, they suggest that metabolic processes in the hippocampus may have an important role in the neurobiology of anxiety.

Translational Psychiatry (2012) 2, e187; doi:10.1038/tp.2012.107; published online 13 November 2012

\section{Introduction}

Mitochondria are intracellular organelles that are involved in numerous essential cellular processes, including the production of cellular energy in the form of ATP through oxidative phosphorylation. ${ }^{1}$ Some of the mitochondrial proteins are produced from the mitochondrial genome, which is maternally inherited. Other proteins essential for mitochondrial function are encoded by the nuclear genome and transported into the mitochondria. Mitochondrial disorders are clinical syndromes produced by mutations in the mitochondrial genome or nuclear genome that result in deficient mitochondrial energy production in highly energy-dependent organs such as the muscle and brain. ${ }^{2}$ Since the brain is the organ of the mind, brain dysfunction can be expected to produce disturbances of mentation, mood and behavior. The majority of the literature has focused on the medical and neurological sequelae of mitochondrial dysfunction. It is becoming increasingly apparent that patients with mitochondrial disorders can also have significant comorbid psychiatric illness. ${ }^{3-5}$ At the same time, the role of mitochondrial dysfunction in the pathophysiology of major psychiatric disorders, such as depression, bipolar disorder and schizophrenia, has begun to receive attention. ${ }^{6-10}$ There is also accumulating evidence for the involvement of candidate brain regions in psychiatric disorders. The caudate nucleus has a central role in cognitive processes and executive functioning and has been implicated in anxiety and mood disorders. ${ }^{11,12}$ The cingulate cortex is felt to be central to the pathophysiology of depression and other mood and anxiety disorders. ${ }^{12-14}$ Likewise, alterations in both activity and volume of the hippocampus have been reported in mood and anxiety disorders. ${ }^{14-17}$

Patients with mitochondrial disorders offer a unique opportunity to study the pathophysiology of psychiatric symptoms in a population with known mitochondrial dysfunction. To date, the metabolic markers associated with psychiatric symptoms in patients with mitochondrial disorders have not been investigated. Proton magnetic resonance spectroscopy ( $\left.{ }^{1} \mathrm{H}-\mathrm{MRS}\right)$ is an imaging technique that allows for noninvasive quantification of brain metabolites in vivo. The aim of this study was to use ${ }^{1} \mathrm{H}-\mathrm{MRS}$ to examine whether psychiatric symptoms in patients with mitochondrial disorders correlate with metabolic indices in brain regions implicated in

\footnotetext{
${ }^{1}$ Department of Psychiatry and Behavioural Neurosciences and Medicine, McMaster University, Hamilton, Ontario, Canada; ${ }^{2}$ Department of Medicine, McMaster University, Hamilton, Ontario, Canada; ${ }^{3}$ Department of Electrical and Computer Engineering, McMaster University, Hamilton, Ontario, Canada and ${ }^{4}$ Department of Pediatrics, McMaster University, Hamilton, Ontario, Canada

Correspondence: Dr RE Anglin, Department of Psychiatry and Behavioural Neurosciences and Medicine, McMaster University, F413-1 Fontbonne Building, St Joseph's Healthcare,50 Charlton Avenue East, Hamilton, Ontario L8N 4A6, Canada.
}

E-mail: rebecca.anglin@gmail.com

Keywords: anxiety; cingulate; hippocampus; mitochondria; metabolites; proton MRS

Received 6 July 2012; revised 6 September 2012; accepted 6 September 2012 
psychiatric illness, especially the caudate, cingulate cortex and hippocampus.

\section{Methods and materials}

Subject selection. In all, 15 patients with a mitochondrial disorder were recruited from the Neuropsychiatry and Neurometabolic Clinics at McMaster University in Hamilton, Ontario, along with 15 age- ( \pm 5 years) and sex-matched healthy controls. The study was approved by the Research Ethics Board of St Joseph's Healthcare Hamilton and Hamilton Health Sciences. All subjects gave informed consent after a full explanation of the study protocol. Subjects were screened for magnetic resonance imaging compatibility and those with magnetic resonance imaging contraindications (for example, pacemaker, neurostimulator, metallic objects in the eye, aneurysm clips) were excluded from the study. In addition, those with significant metallic dental implants were excluded as these significantly diminish the quality of magnetic resonance spectra. All controls were free from medication and had no current symptoms or medical history of a psychiatric, neurological or systemic illness. The diagnosis of a mitochondrial disorder was made using the Thorburn criteria, and only those with definite or probable mitochondrial disease were included in the study. Of the 15 patients, 11 had a definite mitochondrial disorder with identification of the pathogenic mutation or deletion and 4 had a probable mitochondrial disorder with supportive clinical features, histology and enzymology (Table 1).

Psychiatric evaluation. All study participants underwent a detailed psychiatric evaluation, including completion of the Composite International Diagnostic Interview (CIDI), Hamilton Depression Scale (HAM-D), Hamilton Anxiety Scale (HAM-A), Yale-Brown Obsessive Compulsive Scale (YBOCS), Brief Psychiatric Rating Scale (BPRS), Young Mania Rating Scale (YMRS) and Global Assessment of Function (GAF). Assessments were carried out by an experienced rater (psychiatric research nurse). Information on demographics, past psychiatric history, past medical history, medications, current physical symptoms and age at symptom onset was also collected.

MRS procedure. ${ }^{1} \mathrm{H}-\mathrm{MRS}$ data were collected in a single session on the same day as the psychiatric evaluation, using a GE Healthcare 3.0 Tesla Signa HD Magnetic Resonance Imaging system (General Electric Healthcare, Milwaukee, WI, USA) and a standard eight-channel head coil. Each subject underwent high-resolution anatomical scans followed by single voxel ${ }^{1} \mathrm{H}$-MRS of the caudate, cingulate cortex and left hippocampus. Following a three-plane localizer scan, two $T_{1}$-weighted inversion recovery prepared 3D-fSPGR (fast SPoiled GRadient echo) scans were performed to aid in MRS voxel placement: one axial (repetition time $(T R)=7.5 \mathrm{~ms}$, echo time $(T E)=2.1 \mathrm{~ms}$, inversion time $(\mathrm{TI})=450 \mathrm{~ms}$, flip angle $=12^{\circ}$, field of view $(\mathrm{FOV})=240 \mathrm{~mm}$, slice thickness $=2.0 \mathrm{~mm}$, number of scans (phase encoding steps) $\times$ TR $\times$ number of excitation $($ NEX) $=1$, matrix $=192$ $\times 320$ (zero padded to $512 \times 512$ ), 163 slices) and the other sagittal $\left(T R=10.1 \mathrm{~ms}, \mathrm{TE}=2 \mathrm{~ms}\right.$, flip angle $=20^{\circ}$,
$\mathrm{FOV}=220 \mathrm{~mm}, \quad$ slice $\quad$ thickness $=1.2 \mathrm{~mm}, \quad \mathrm{NEX}=2$, matrix $=192 \times 320$ (zero padded to $512 \times 152), \quad 123$ images). In addition, an axial $\mathrm{T}_{2}$ fluid attenuation inversion recovery scan $(T R=9000 \mathrm{~ms}, T E=139.8 \mathrm{~ms}, F O V=220$ $\mathrm{mm}$, matrix $=192 \times 320$ (zero padded to $512 \times 512) 25$ slices $5.0 \mathrm{~mm}$ thick, $\mathrm{NEX}=2$ ) was acquired to aid in ruling out gross neuropathology.

A standardized positioning procedure was used to place voxels in the caudate, cingulate cortex and hippocampus (Figure 1). Voxel size was $20 \times 20 \times 20 \mathrm{~mm}^{3}$. The caudate voxel was initially placed using the sagittal series and centered on the left caudate. The axial anatomical series were then used to adjust the voxel to include maximal caudate volume. The cingulate cortex voxel was placed using the sagittal and axial scans, adjacent to the corpus callosum on the left side. The hippocampal voxel was first situated on the sagittal anatomic scan and centered on the head of the left hippocampus, and then adjusted to be adjacent to the medial aspect of the lateral fissure using the axial anatomical series.

Single voxel ${ }^{1} \mathrm{H}-M R S$ was conducted using a short echo PRESS (Point RESolved Spectroscopy) sequence (TE $=35$ $\mathrm{ms}, \mathrm{TR}=2000 \mathrm{~ms}, 256$ acquisitions). Voxel shimming was performed to second order. The MRS protocol took approximately $60 \mathrm{~min}$ in total. Tissue segmentation was performed using a combination of in-house developed software and the freeware package (Analysis of Functional Neurolmages). ${ }^{18}$ Using high-resolution axial anatomical images, white matter (WM) and cerebrospinal fluid (CSF) masks were created to determine the gray-scale intensity of each tissue (gray matter (GM) was taken to be the intensities between WM and CSF). MRS voxel masks were then segmented using these intensity values to calculate the fraction of GM, WM and CSF within each voxel. Spectral analysis was carried out using LCModel. $^{19}$ LCModel is an operator-independent software that performs automatic quantification of in vivo proton magnetic resonance spectra. Partial volume correction was performed according to LCModel. ${ }^{19,20}$ Total water concentration for each volume of interest was adjusted using the equation: $w_{\text {conc }}=43$ $300 f_{\mathrm{gm}}+35880 f_{\mathrm{wm}}+55556 f_{\mathrm{csf}}$, where $f_{\mathrm{gm}}, f_{\mathrm{wm}}$ and $f_{\mathrm{csf}}$ are the fraction of GM, WM and CSF tissue within each voxel. Water concentrations in GM, WM and CSF were taken from Ernst et al. ${ }^{21}$ As metabolites are concentrated in only the GM and WM, the final concentration was then divided by: $1-f_{\mathrm{csf}}$. A sample spectrum from a mitochondrial patient is presented in Figure 2. A Cramér-Rao error of less than $20 \%$ was used as a cutoff for inclusion in the analysis. No data was excluded based on this cutoff. The mean signal-to-noise ratio \pm s.d. was $16.27 \pm 2.89$ for the caudate, $17.27 \pm 4.38$ for the cingulate and $20.53 \pm 3.09$ for the hippocampus for the mitochondrial patients. The mean full-width at half-maximum \pm s.d. was $0.09 \pm 0.02$ for the caudate, $0.09 \pm 0.03$ for the cingulate and $0.08 \pm 0.01$ for the hippocampus. Institutional concentrations of $\mathrm{N}$-acetyl aspartate, creatine $(\mathrm{Cr})$, glycerophosphocholine (GPC), myoinositol and glutamate + glutamine (GIx) for each region of interest were derived.

Statistical analysis. Statistical analyses were performed with PASW Statistics 18.0 (SPSS, Chicago, IL, USA). Twotailed significance was assessed at the $P<0.05$ threshold. An independent sample $t$-test was performed to compare 


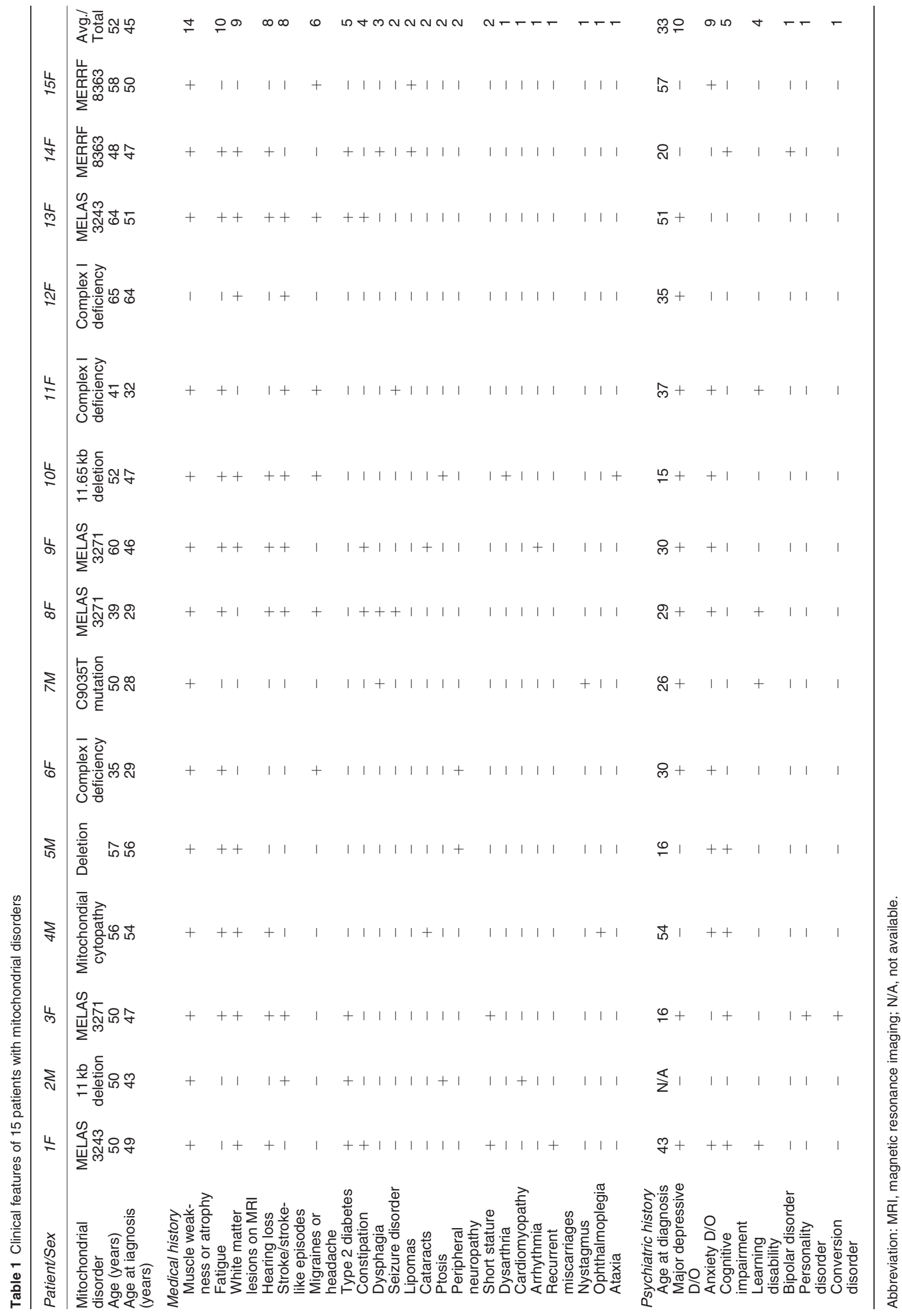


mean scores on the psychiatric rating scales between patients and controls. Only scales showing a significant difference (HAM-A, HAM-D and GAF) were used for the
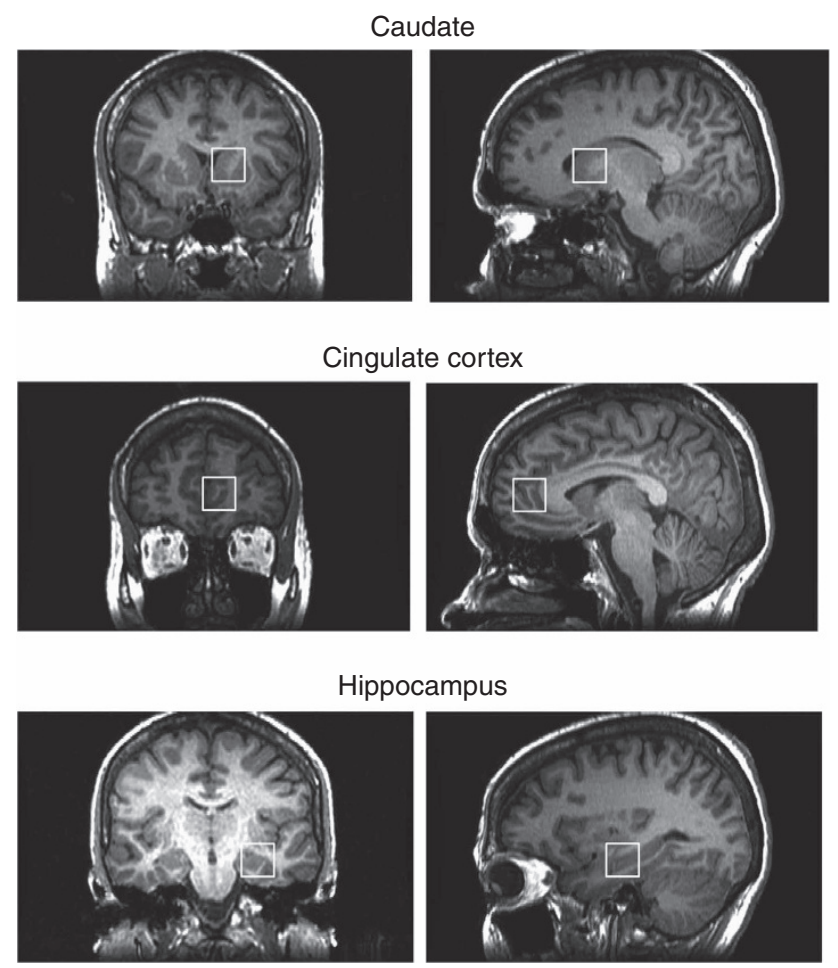

Figure 1 Representative voxel placement for proton magnetic resonance spectroscopy ('H-MRS) acquisition. correlational analyses. Pearson's correlation coefficients were used to determine correlations between metabolites and the psychiatric rating scales for the 15 mitochondrial disease patients. A Holm-Bonferroni correction for multiple comparisons was used for each region of interest. Linear regression was also performed to determine the coefficients of determination $\left(R^{2}\right)$ values for each rating scale regressed onto each metabolite for the three region of interests.

\section{Results}

Demographics and clinical data. Patients with mitochondrial disorders and healthy controls were pair-matched by age (mitochondrial disorder 51.7 \pm 8.7 , healthy controls 50.2 $\pm 8.6, P=0.65)$ and sex (11 F/4 $\mathrm{M}$ in each group). Clinical and demographic information for the 15 patients with mitochondrial disorders is presented in Table 1. The average age at diagnosis of a mitochondrial disorder was 45 years. Of the 15 patients, 14 had a history of a psychiatric illness, some with multiple diagnoses, including major depressive disorder in 10 patients, anxiety disorder in 9 and bipolar disorder, personality disorder and conversion disorder each in 1 patient. The average age at diagnosis of a psychiatric disorder was 33 years, and the diagnosis of a psychiatric illness preceded the diagnosis of a mitochondrial disorder in the majority of patients. Four patients had a history of learning disability, and five had cognitive impairment. The most common physical features were muscle weakness or atrophy, fatigue, hearing loss, stroke-like episodes, migraine and Type 2 diabetes. Table 2 describes the psychotropic medications and supplements used by the mitochondrial patients. Of the fifteen patients, 13 were treated with mitochondrial supplements, including creatine monohydrate,

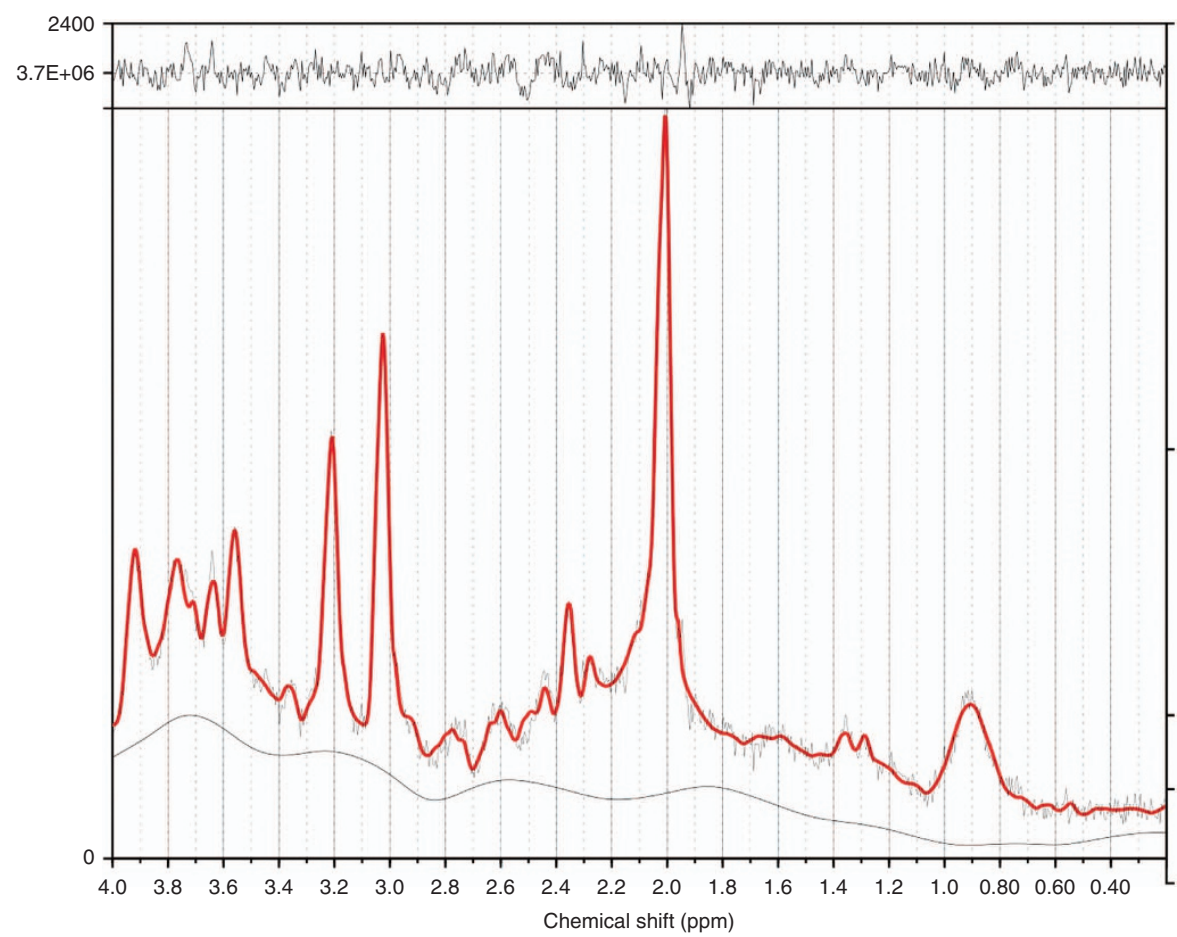

Figure 2 Representative magnetic resonance spectroscopy (MRS) spectrum from the cingulate cortex of a mitochondrial patient. 
Table 2 Mitochondrial supplements and psychotropic medications used by 15 patients with mitochondrial disorders

\begin{tabular}{|c|c|c|c|c|c|c|c|c|c|c|c|c|c|c|c|}
\hline Patient/sex & $1 F$ & $2 M$ & $3 F$ & $4 M$ & $5 M$ & $6 F$ & $7 M$ & $8 F$ & $9 F$ & $10 F$ & $11 F$ & $12 F$ & $13 F$ & $14 F$ & $15 F$ \\
\hline \multicolumn{16}{|c|}{ Mitochondrial supplements } \\
\hline Creatine & + & + & + & + & + & + & + & + & + & + & - & + & + & + & - \\
\hline$\alpha$-Lipoic acid & + & + & + & + & + & + & + & + & + & + & - & - & + & + & - \\
\hline Riboflavin & + & + & + & + & + & + & + & + & + & + & - & - & + & + & - \\
\hline Vitamin C & + & + & + & + & + & + & + & + & + & + & - & - & + & + & - \\
\hline Vitamin E & + & + & + & + & + & + & + & + & + & + & - & - & + & + & - \\
\hline Coenzyme Q10 & + & + & + & + & + & + & + & + & + & + & - & + & + & + & - \\
\hline \multicolumn{16}{|c|}{ Psychotropic medications } \\
\hline Benzodiazepine & + & - & - & + & - & - & - & - & - & - & - & - & - & - & - \\
\hline Fluvoxamine & + & - & - & - & - & - & - & - & - & - & - & - & - & - & - \\
\hline Fluoxetine & - & - & - & - & - & + & - & - & - & - & - & - & - & - & - \\
\hline Amitriptyline & - & - & - & - & + & - & - & - & - & - & - & - & - & - & - \\
\hline Olanzapine & - & - & - & - & - & - & - & - & - & - & - & - & - & + & - \\
\hline Valproic acid & - & - & - & - & - & - & - & - & - & - & - & - & - & + & - \\
\hline
\end{tabular}

Table 3 Psychiatric rating scales in 15 mitochondrial patients and 15 controls

\begin{tabular}{lccc}
\hline & $\begin{array}{c}\text { Mitochondrial patients } \\
\text { (mean } \pm \text { s.d.) }\end{array}$ & $\begin{array}{c}\text { Controls } \\
\text { (mean } \pm \text { s.d.) }\end{array}$ & P-value \\
\hline Age & $51.7 \pm 8.7$ & $50.2 \pm 8.6$ & 0.65 \\
(years) & $71.7 \pm 7.0$ & $90.7 \pm 4.4$ & 0.0001 \\
GAF & $10.7 \pm 5,4$ & $1.8 \pm 2.6$ & 0.0001 \\
HAM-D & $9.4 \pm 3.5$ & $2.1 \pm 3.1$ & 0.0001 \\
HAM-A & $1.9 \pm 5.0$ & $1.1 \pm 3.1$ & 0.65 \\
YBOC & $27.1 \pm 5.0$ & $25.4 \pm 2.4$ & 0.24 \\
BPRS & $0.5 \pm 1.2$ & $1.1 \pm 2.0$ & 0.29 \\
YMRS & & & \\
\hline
\end{tabular}

Abbreviations: BPRS, Brief Psychiatric Rating Scale; GAF, Global Assessment of Function; HAM-A, Hamilton Anxiety Scale; YBOC, Yale-Brown Obsessive Compulsive Scale; YMRS, Young Mania Rating Scale.

and 5 of the 15 patients were using psychotropic drugs, including benzodiazepines, selective serotonin reuptake inhibitor antidepressants, tricyclic antidepressants, the atypical antipsychotic olanzapine and the mood stabilizer valproic acid. None of the healthy controls was receiving any mitochondrial supplements or psychotropic medications. Patients had significantly lower GAF scores compared with controls $(P=0.0001)$ and significantly higher HAM-D $(P=0.0001)$ and HAM-A scores $(P=0.0001)$. There were no differences in YBOCS, BPRS or YMRS scores (Table 3).

Correlation between metabolites and psychiatric rating scales. Significant correlations were identified between HAM-A scores and $\mathrm{Cr}$ (Pearson's correlation coefficient $(\mathrm{PCC})=0.64, \quad P=0.011), \quad \mathrm{GPC} \quad(\mathrm{PCC}=0.66, P=0.008)$, myoinositol $(P C C=0.58, P=0.02)$ and $\mathrm{Glx}(\mathrm{PCC}=0.60$, $P=0.02)$ in the hippocampus. GAF scores showed a significant correlation with $\mathrm{GPC}(\mathrm{PCC}=-0.55, P=0.03)$ and Glx (PCC $=-0.67, P=0.01)$ in the cingulate cortex (Table 4). After Holm-Bonferroni correction for multiple comparisons, the correlation between HAM-A and GPC levels in the hippocampus remained significant, as did the correlation between HAM-A and $\mathrm{Cr}$. The correlations between GAF and GIx levels in the cingulate cortex likewise remained significant after Holm-Bonferroni correction. The corresponding scatter plots and regression lines for the significant associations between psychiatric rating scales and metabolites are presented in Figure 3. The $R^{2}$ values ranged from 0.30 to 0.45 , indicating moderate correlation. There were no significant correlations between metabolites and psychiatric rating scales in the caudate.

\section{Discussion}

To our knowledge, this is the first study to look at the neuroimaging correlates of psychiatric symptoms in patients with mitochondrial disorders. Despite burgeoning interest in the potential involvement of mitochondria in the neurobiology of psychiatric illness, ${ }^{5}$ there has been relatively less attention paid to the psychiatric dimensions of mitochondrial disorders compared with the medical and neurological sequelae. ${ }^{22}$ Patients with mitochondrial disorders offer a unique window through which we can begin to understand the association between psychiatric symptoms and mitochondrial dysfunction in vivo. Using ${ }^{1} \mathrm{H}-\mathrm{MRS}$, we were able to investigate metabolic indices in mitochondrial patients in regions of the brain that have been implicated in psychiatric illness: the caudate, cingulate cortex and hippocampus. We found that anxiety symptoms in these patients correlated with higher levels of multiple metabolic markers in the hippocampus including a significant positive correlation with levels of GPC and Cr. Impaired overall level of function as a result of psychiatric symptoms (measured using the GAF) correlated with metabolite levels in the cingulate cortex, including a significant relationship with Glx. These findings suggest that metabolic processes in the hippocampus and cingulate may have a role in producing the psychiatric symptoms experienced by these patients.

How might metabolic markers in the hippocampus and cingulate contribute to the development of neuropsychiatric symptoms?. The hippocampus was the only brain region in which metabolic measures correlated with anxiety symptoms (Table 4). Recent evidence points to a central role for the hippocampus in the neurobiology of anxiety. Neurogenesis in the dentate gyrus of the hippocampus has been found to play a crucial role in allowing adaptation to different environments, by shifting the balance between the differentiating of similar inputs (pattern separation) and the combining of different inputs into a complete representation 

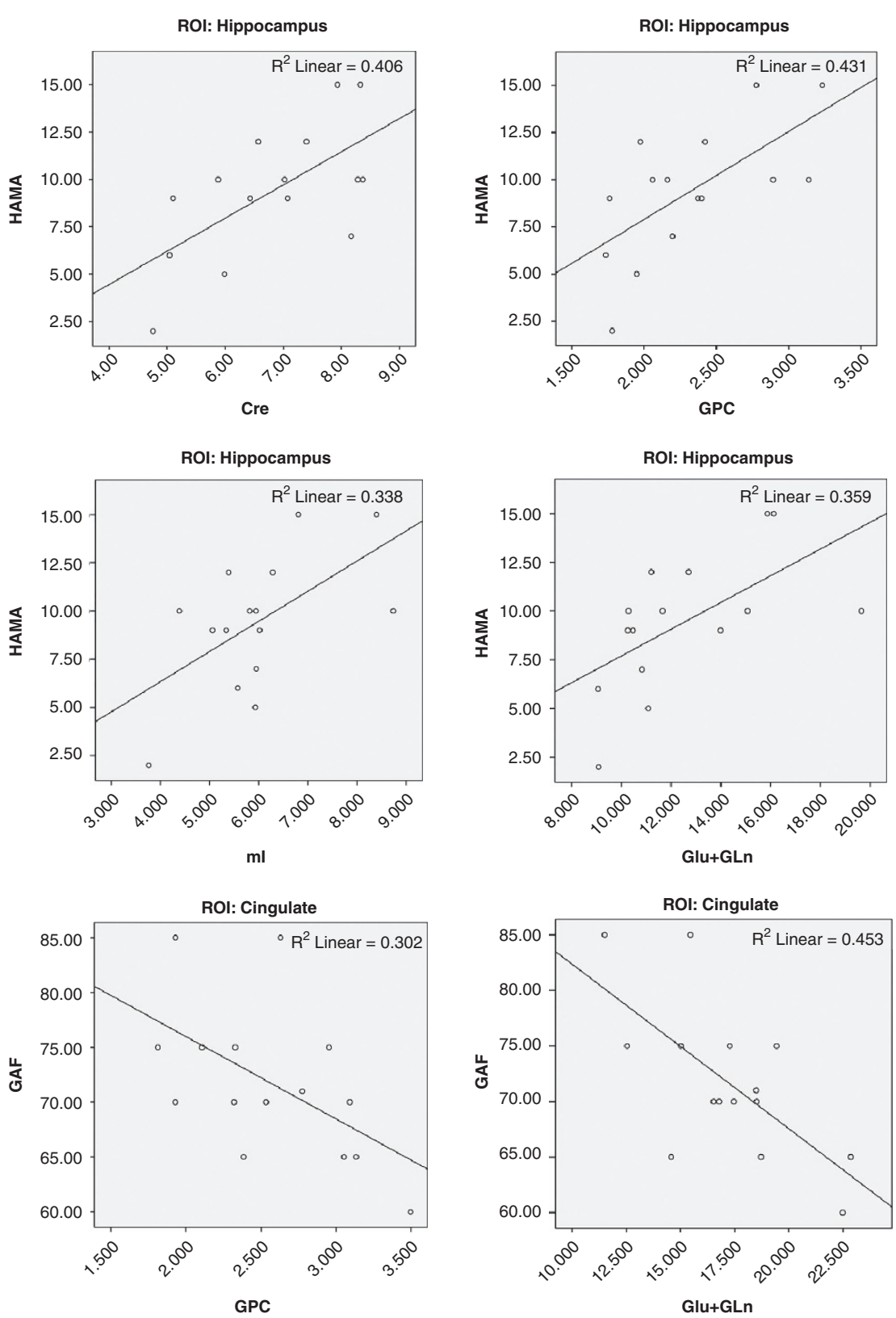

Figure 3 Scatter plots of correlations between metabolites and psychiatric rating scales for 15 mitochondrial patients.

(pattern completion). ${ }^{23}$ Stressful environments lead to decreased hippocampal neurogenesis, and decreased pattern separation, resulting in inability to properly distinguish nonthreatening new situations from earlier anxiogenic episodes. ${ }^{23}$ This generalizing of a particular anxiety-provoking experience to otherwise-innocuous new situations leads to what we know as generalized anxiety disorder. By contrast, increased hippocampal neurogenesis results in improved pattern separation and decreased anxiety behavior. ${ }^{24}$ Therapeutic interventions such as exercise and selective serotonin reuptake inhibitor antidepressants appear to increase neurogenesis and synaptic plasticity in the hippocampus. ${ }^{25}$ When combined with extinction training, this results in remodeling of the fear memory circuit, and extinction of anxiety-related behavior. ${ }^{25}$

The correlation we observed between impaired global functioning, as a result of psychiatric symptoms, and metabolic markers in the cingulate cortex is consistent with previous findings that the cingulate as a key component of the limbic system, is involved in the neurobiology of psychiatric illness. ${ }^{26}$ We had anticipated that there might be a correlation between depressive symptoms and metabolite levels in this region, given the evidence pointing to cingulate involvement in the pathobiology of depression. ${ }^{27-29}$ These studies, however, tended to involve patients with severe and treatment-resistant depression, with one study reporting a 
Table 4 Correlations between selected psychiatric rating scales and metabolites by region, expressed as Pearson's correlation coefficients $(r)$ and corresponding $P$-values

\begin{tabular}{|c|c|c|c|}
\hline Metabolites & $\begin{array}{c}\text { HAM-A } \\
(\mathrm{r}, \mathrm{P} \text {-value) }\end{array}$ & $\begin{array}{c}G A F \\
(\mathrm{r}, \mathrm{P}-\text { value })\end{array}$ & $\begin{array}{c}H A M-D \\
(\mathrm{r}, \mathrm{P}-\text {-value })\end{array}$ \\
\hline \multicolumn{4}{|l|}{ Caudate } \\
\hline NAA & $0.247,0.374$ & $-0.198,0.48$ & $0.452,0.091$ \\
\hline $\mathrm{Cr}$ & $0.031,0.913$ & $-0.40,0.15$ & $-0.004,0.988$ \\
\hline GPC & $0.122,0.665$ & $-0.46,0.09$ & $0.147,0.601$ \\
\hline $\mathrm{ml}$ & $0.257,0.355$ & $0.31,0.27$ & $-0.056,0.844$ \\
\hline Glx & $0.157,0.575$ & $-0.23,0.41$ & $0.181,0.519$ \\
\hline \multicolumn{4}{|c|}{ Hippocampus } \\
\hline NAA & $0.36,0.19$ & $0.37,0.17$ & $0.00,0.99$ \\
\hline $\mathrm{Cr}$ & $0.64,0.01^{a}$ & $0.17,0.55$ & $0.23,0.41$ \\
\hline GPC & $0.66,0.01^{a}$ & $0.05,0.85$ & $0.36,0.18$ \\
\hline $\mathrm{ml}$ & $0.58,0.02^{b}$ & $0.15,0.60$ & $0.35,0.21$ \\
\hline Glx & $0.60,0.02^{b}$ & $0.01,0.97$ & $0.38,0.17$ \\
\hline \multicolumn{4}{|c|}{ Cingulate cortex } \\
\hline NAA & $0.10,0.73$ & $-0.20,0.47$ & $0.32,0.25$ \\
\hline $\mathrm{Cr}$ & $-0.43,0.11$ & $-0.51,0.06$ & $-0.22,0.43$ \\
\hline GPC & $-0.20,0.48$ & $-0.55,0.03^{b}$ & $-0.14,0.62$ \\
\hline $\mathrm{ml}$ & $-0.03,0.91$ & $-0.05,0.87$ & $0.13,0.64$ \\
\hline Glx & $-0.24,0.39$ & $-0.67,0.01^{a}$ & $0.06,0.83$ \\
\hline
\end{tabular}

Abbreviations: Cr, creatinine; GAF, Global Assessment of Function; Glx glutamate + glutamine; GPC, glycerophosphocholine; HAM-A, Hamilton Anxiety Scale; HAM-D, Hamilton Depression Scale; ml, myoinositol; NAA, N-acetyl aspartate.

remains significant after Holm-Bonferroni correction for multiple comparisons. ${ }^{\mathrm{b}} P$-value $<0.05$

mean HAM-D score of $23.9,{ }^{29}$ whereas the mean HAM-D scores for those in our series was only 10.7 , consistent with mild depression. ${ }^{30}$ It is likely that the depressive symptoms in our patients were simply too mild to result in detectable correlations.

What is the significance of the individual metabolic markers in relation to psychiatric symptoms?. (1) Increased glutamatergic activity $(\uparrow G / x)$ : We found a significant correlation between measures of psychiatric symptomatology and levels of Glx in the cingulate cortex. The Glx peak measured using ${ }^{1} \mathrm{H}$-MRS is a composite peak, composed primarily of glutamate and glutamine. ${ }^{31}$ Glutamate is the most abundant amino acid in the brain, and an important excitatory neurotransmitter, ${ }^{31}$ acting on a multiplicity of receptors including $N$-methyl-D-aspartic acid, AMPA/ kainate and metabotropic families. Glutamate is removed from the synapse through an active transporter system and converted in astrocytes to glutamine by glutamine synthetase. When glutamine is then released from the astrocytes, it is taken up by neurons and converted back to glutamate by phosphate-activated glutaminase. Glutamate can also be converted to $\gamma$-amino butyric acid (GABA), the major inhibitory neurotransmitter of the brain, in a bidirectional manner. In addition, glutamate can be synthesized de novo from Krebs's cycle intermediates.

The exact meaning of the combined Glx peak remains unclear. Although this measure is generally interpreted as reflecting glutamatergic activity and excitotoxicity, this may not be entirely accurate. ${ }^{32}$ If the Glx peak reflects the total pool of glutamate and glutamine, expansion of this pool would appear to require increased synthesis of glutamate through Kreb's cycle intermediates, or decreased degradation, or a shift in the glutamate-GABA shuttle in favor of glutamate. This last-mentioned possibility is intriguing; if the increase in Glx reflects a decrease in GABA, this might be an explanation for the correlation we observed between higher GIx and measures of psychiatric symptomatlogy. Decreased inhibitory signaling by GABA has been demonstrated in patients with anxiety disorders. ${ }^{33}$ The role of GABA in anxiety is further supported by the therapeutic efficacy of benzodiazepines, which enhance GABA neurotransmission by promoting chloride conductance at the $\mathrm{GABA}_{\mathrm{A}}$ receptor.

Previous observations of increased Glx in patients with mitochondrial disorders have been taken to indicate increased glutamatergic excitotoxicity. ${ }^{34}$ Mitochondria, as the 'power plants' of the cell, generate the majority of cellular energy through oxidative phosphorylation. Mitochondrial dysfunction, therefore, causes an energy deficit that can result in a failure of many cellular mechanisms, including function of the energy-dependent sodium-potassium pumps. Failure of these pumps would result in a decreased threshold for membrane depolarization and increased glutamate release from presynaptic terminals. At the same time, reduced energy may cause failure of glutamate reuptake, further increasing the concentration of glutamate in the synapse. Extracellular glutamate will stimulate the kainate receptor, causing sodium influx and a further decrease in membrane potential. This will release the magnesium blockade on $N$-methyl-D-aspartic acid receptors, resulting in calcium entry into the cell and triggering of the excitotoxic process, including activation of the apoptotic cascade. If the Glx peak is indeed a reflection of excitotoxic activity, this might explain how impairment of overall function in our patients could be related to higher levels of Glx in the cingulate cortex.

(2) Increased creatine ( $\uparrow \mathrm{Cr}$ ): Cr levels in the hippocampus were significantly correlated with anxiety symptoms in our patients. The $\mathrm{Cr}$ peak in ${ }^{1} \mathrm{H}-\mathrm{MRS}$ is a composite, composed of both $\mathrm{Cr}$ and phosphocreatine. Phosphocreatine is a highenergy compound that acts as a reservoir to generate energy through the donation of a phosphate group to ADP, resulting in $\mathrm{Cr}$ and ATP. ${ }^{35}$ It is difficult to interpret the significance of the elevated $\mathrm{Cr}$ peak in ${ }^{1} \mathrm{H}$-MRS as this may reflect an increase in $\mathrm{Cr}$, phosphocreatine or both. Of interest is the fact that most patients in this study were receiving $\mathrm{Cr}$ supplementation, which may have elevated their $\mathrm{Cr}$ levels. ${ }^{36} \mathrm{Cr}$ supplementation has anecdotally been suggested to increase anxiety symptoms. ${ }^{37}$

(3) Increased membrane turnover ( $\uparrow$ GPC): There was also a significant correlation in our study between anxiety symptoms and hippocampal levels of GPC. GPC is a breakdown product of phosphatidylcholine and is therefore elevated in situations of phospholipid breakdown and membrane degradation. With failure of oxidative phosphorylation as a result of mitochondrial dysfunction, catabolic enzymes such as phospholipases may be activated through apoptotic pathways, resulting in elevated GPC. ${ }^{38}$ If higher levels of GPC can be taken to reflect hippocampal dysfunction, this could explain the association with anxiety symptoms that we observed. 


\section{Future directions}

We were able to identify several significant correlations, even after correction for multiple comparisons, despite having a relatively small sample size. Additional trends for some metabolites were identified in the hippocampus and cingulate that are intriguing and would benefit from further investigation in a larger sample size. Our findings suggest that metabolic markers in the hippocampus and cingulate are associated with psychiatric symptoms, particularly anxiety, in patients with mitochondrial disorders. Future studies of these metabolic indices in patients with anxiety alone will help clarify whether the identified correlations are limited to those with mitochondrial disorders, or whether they are relevant to the pathophysiology of anxiety in general. To date there have been very few ${ }^{1} \mathrm{H}$-MRS studies of patients with generalized anxiety disorder, and none that have comprehensively measured metabolic indices in these regions of interest.

\section{Conclusions}

Anxiety correlated specifically with metabolic indices in the hippocampus and impairment of overall functioning as a result of psychiatric symptoms correlated with levels in the cingulate cortex, measured using ${ }^{1} \mathrm{H}-\mathrm{MRS}$, in patients with mitochondrial disorders. These results are of particular interest in light of recent evidence implicating the hippocampus in the pathobiology of anxiety. Our findings lend support to the notion that metabolic abnormalities in these regions, potentially associated with mitochondrial dysfunction, may have a role in the pathophysiology of psychiatric illness.

\section{Conflict of interest}

Dr Tarnopolsky has received speaker honoraria from Transgenomics for talks on mitochondrial disease in 2009. The remaining authors declare no conflict of interest.

Acknowledgements. Funding for this project was provided by a peerreviewed grant from Physicians Services Incorporated (PSI Grant R0624). Dr Anglin is supported by an Ontario Mental Health Foundation (OMHF) Research Training Fellowship Award. We thank Alexander Weber for his assistance with tissue segmentation.

1. Wallace DC. Mitochondrial diseases in man and mouse. Science 1999; 283: $1482-1488$.

2. van Adel BA, Tarnopolsky MA. Metabolic myopathies: update 2009. J Clin Neuromuscul Dis 2009; 10: 97-121.

3. Anglin RE, Garside SL, Tarnopolsky MA, Mazurek MF, Rosebush PI. The psychiatric manifestations of mitochondrial disorders: a case and review of the literature. J Clin Psychiatry 2012; 73: 506-512.

4. Anglin R, Tarnopolsky MA, Mazurek MF, Rosebush PI. The psychiatric presentation of mitochondrial disorders in adults. J Neuropsychiatr Clin Neurosc 2012; 24: 1-16.

5. Anglin RE, Mazurek MF, Tarnopolsky MA, Rosebush PI. The mitochondrial genome and psychiatric illness. Am J Med Genet B Neuropsychiatr Genet 2012; 159B: 749-759.

6. Hough CJ, Chuang DM. The mitochondrial hypothesis of bipolar disorder. Bipolar Disord 2000; 2(Part 1): 145-147.

7. Kato T. The role of mitochondrial dysfunction in bipolar disorder. Drug News Perspect 2006; 19: 597-602.

8. BenShachar D, Laifenfeld D. Mitochondria, synaptic plasticity, and schizophrenia. Int Rev Neurobiol 2004; 59: 273-296.

9. Burnett BB, Gardner A, Boles RG. Mitochondrial inheritance in depression, dysmotility and migraine? J Affect Disord 2005; 88: 109-116.

10. Young LT. Is bipolar disorder a mitochondrial disease? J Psychiatry Neurosci 2007; 32: 160-161.
11. Grahn JA, Parkinson JA, Owen AM. The cognitive functions of the caudate nucleus. Prog Neurobiol 2008; 86: 141-155.

12. Holzschneider K, Mulert C. Neuroimaging in anxiety disorders. Dialog Clin Neurosci 2011; 13: 453-461.

13. Ressler KJ, Mayberg HS. Targeting abnormal neural circuits in mood and anxiety disorders: from the laboratory to the clinic. Nat Neurosci 2007; 10: 1116-1124.

14. Etkin A, Wager TD. Functional neuroimaging of anxiety: a metaanalysis of emotional processing in PTSD, social anxiety disorder, and specific phobia. Am J Psychiatry 2007; 164: 1476-1488.

15. Campbell S, Marriott M, Nahmias C, MacQueen GM. Lower hippocampal volume in patients suffering from depression: a metaanalysis. Am J Psychiatry 2004; 161: 598-607.

16. Sheline YI. Hippocampal atrophy in major depression: a result of depressioninduced neurotoxicity? Mol Psychiatry 1996; 1: 298-299.

17. Gilbertson MW, Shenton ME, Ciszewski A, Kasai K, Lasko NB, Orr SP et al. Smaller hippocampal volume predicts pathologic vulnerability to psychological trauma. Nat Neurosci 2002; 5: 1242-1247.

18. Cox RW. AFNl: software for analysis and visualization of functional magnetic resonance neuroimages. Comput Biomed Res 1996; 29: 162-173.

19. Provencher SW. Estimation of metabolite concentrations from localized in vivo proton NMR spectra. Magn Reson Med 1993; 30: 672-679.

20. LC Model Manual. http://lcmodel.ca/pages/lcm-manual.shtml, 2011.

21. Ernst $T$, Kreis R, Kreis R. Absolute quantitation of water and metabolites in the human brain. 1. Compartments and water. J Magn Reson Ser B 1993; 102: 1064-1866.

22. Anglin R, Garside S, Tarnopolsky MA, Mazurek MF, Rosebush PI. The psychiatric manifestations of mitochondrial disorders: a case and review of the literature. J Clin Psychiatry 2012; 73: 506-512.

23. Sahay A, Wilson DA, Hen R. Pattern separation: a common function for new neurons in hippocampus and olfactory bulb. Neuron 2011; 70: 582-588.

24. Sahay A, Scobie KN, Hill AS, O'Carroll CM, Kheirbek MA, Burghardt NS et al. Increasing adult hippocampal neurogenesis is sufficient to improve pattern separation. Nature 2011; 472: 466-470.

25. Karpova NN, Pickenhagen A, Lindholm J, Tiraboschi E, Kulesskaya N, Agustsdottir A et al. Fear erasure in mice requires synergy between antidepressant drugs and extinction training. Science 2011; 334: 1731-1734.

26. Yucel M, Wood SJ, Fornito A, Riffkin J, Velakoulis D, Pantelis C. Anterior cingulate dysfunction: implications for psychiatric disorders? J Psychiatry Neurosci 2003; 28: 350-354.

27. Hamani C, Mayberg H, Stone S, Laxton A, Haber S, Lozano AM. The subcallosal cingulate gyrus in the context of major depression. Biol Psychiatry 2011; 69: 301-308.

28. Mayberg HS, Brannan SK, Mahurin RK, Jerabek PA, Brickman JS, Tekell JL et al. Cingulate function in depression: a potential predictor of treatment response. NeuroReport 1997; 8: 1057-1061.

29. Holtzheimer PE, Kelley ME, Gross RE, Filkowski MM, Garlow SJ, Barrocas A et al. Subcallosal cingulate deep brain stimulation for treatmentresistant unipolar and bipolar depression. Arch Gen Psychiatry 2012; 69: 150-158.

30. Hamilton M. A rating scale for depression. J Neurol Neurosurg Psychiatry 1960; 23: 56-62.

31. Barker PB, Bizzi A, De Stefano N, Gullapalli R, Lin DDM. Clinical MR Spectroscopy Techniques and Applications. 1st edn. Cambridge University Press: Cambridge, 2010.

32. Yuksel C, Ongur D. Magnetic resonance spectroscopy studies of glutamaterelated abnormalities in mood disorders. Biol Psychiatry 2010; 68: 785-794.

33. Martin El, Ressler KJ, Binder E, Nemeroff CB. The neurobiology of anxiety disorders: brain imaging, genetics, and psychoneuroendocrinology. Clin Lab Med 2010; 30: 865-891.

34. Mark LP, Prost RW, Ulmer JL, Smith MM, Daniels DL, Strottmann JM et al. Pictorial review of glutamate excitotoxicity: fundamental concepts for neuroimaging. Am J Neuroradiol 2001; 22: 1813-1824.

35. Andres RH, Ducray AD, Schlattner U, Wallimann T, Widmer HR. Functions and effects of creatine in the central nervous system. Brain Res Bull 2008; 76: 329-343.

36. Lyoo IK, Kong SW, Sung SM, Hirashima F, Parow A, Hennen J et al. Multinuclear magnetic resonance spectroscopy of highenergy phosphate metabolites in human brain following oral supplementation of creatinemonohydrate. Psychiatry Res 2003; 123: 87-100.

37. Poortmans JR, Francaux M. Adverse effects of creatine supplementation: fact or fiction? Sports Med 2000; 30: 155-170.

38. Klein J. Membrane breakdown in acute and chronic neurodegeneration: focus on cholinecontaining phospholipids. J Neural Transm 2000; 107: 1027-1063.

Translational Psychiatry is an open-access journal published by Nature Publishing Group. This work is licensed under the Creative Commons Attribution-NonCommercial-No Derivative Works 3.0 Unported License. To view a copy of this license, visit http://creativecommons.org/licenses/by-nc-nd/3.0/ 